SAND98-1272

Unlimited Release

Printed June 1998

\title{
Final Report on LDRD Project: The Double Electron Layer Tunneling Transistor (DELTT)
}

\author{
J. A. Simmons, J. S. Moon, M. A. Blount, W. E. Baca, and N. E. Harff \\ Nanostructure and Semiconductor Physics \\ S. K. Lyo and J. L. Reno \\ Semiconductor Material and Device Sciences \\ J. R. Wendt, M. E. Sherwin, M. V. Weckwerth, M. J. Hafich, and J. F. Klem \\ Semiconductor Materials and Processes \\ Sandia National Laboratories \\ P.O. Box 5800 \\ Albuquerque, New Mexico 87185-1415
}

\begin{abstract}
This report describes the research accomplishments achieved under the LDRD Project "Double Electron Layer Tunneling Transistor." The main goal of this project was to investigate whether the recently discovered phenomenon of 2D-2D tunneling in GaAs/AlGaAs double quantum wells (DQWs), investigated in a previous LDRD, could be harnessed and implemented as the operating principle for a new type of tunneling device we proposed, the double electron layer tunneling transistor (DELTT). In parallel with this main thrust of the project, we also continued a modest basic research effort on DQW physics issues, with significant theoretical support.

The project was a considerable success, with our main goal of demonstrating a working prototype of the DELTT having been achieved. Additional DELTT advances included demonstrating good electrical characteristics at $77 \mathrm{~K}$, demonstrating both NMOS and CMOS-like bi-stable memories at $77 \mathrm{~K}$ using the DELTT, demonstrating digital logic gates at $77 \mathrm{~K}$, and demonstrating voltage-controlled oscillators at $77 \mathrm{~K}$. In order to successfully fabricate the DELTT, we had to develop a novel flip-chip processing scheme, the epoxy-bond-and-stop-etch (EBASE) technique. This technique was later improved so as to be amenable to electron-beam lithography, allowing the fabrication of DELTTs with sub-micron features, which are expected to be extremely high speed. In the basic physics area we also made several advances, including a measurement of the effective mass of electrons in the hour-glass orbit of a DQW subject to in-plane magnetic fields, and both measurements and theoretical calculations of the full Landau level spectra of DQWs in both perpendicular and in-plane magnetic fields. This last result included the unambiguous demonstration of magnetic breakdown of the Fermi surface.

Finally, we also investigated the concept of a far-infrared photodector based on photon assisted tunneling in a DQW. Absorption calculations showed a narrowband absorption which persisted to temperatures much higher than the photon energy being detected. Preliminary data on prototype detectors indicated that the absorption is not only narrowband, but can be tuned in energy through the application of a gate voltage.




\section{Table of Contents}

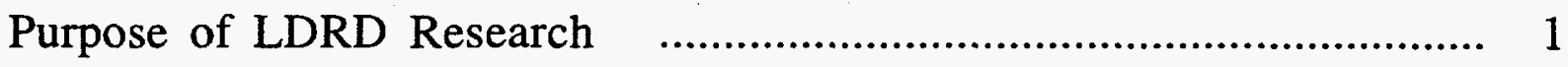

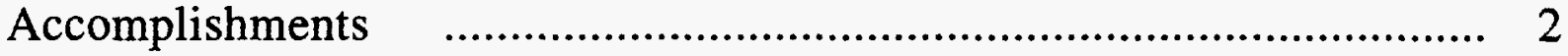

1. Development of the EBASE flip-chip processing technique $\ldots . . . . . . . . . . . .2$

2. Initial demonstration of transistor action in a DELTT at $\mathrm{T} \leq 4.2 \mathrm{~K} \quad \ldots \ldots \ldots .3$

3. Demonstration of bistable memory circuits ................................. 3

4. Design and fabrication of higher temperature DELTTs and demonstration

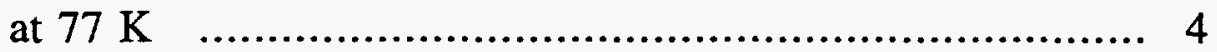

5. Demonstration of digital logic circuits and oscillators $\quad$....................... 4

6. Extension of EBASE technique to encompass electron-beam

lithography

7. Cyclotron mass of lower energy branch in a DQW subject to in-plane magnetic fields

8. Magnetic breakdown and complete DQW Landau level spectrum:

experiment

9. Magnetic breakdown and complete DQW Landau level spectrum:

theory

10. Tunnel-coupled quantum point contacts in split-gate defined double quantum wells

Appendix I. List of Refereed Publications and Presentations

Refereed Publications

Presentations

Xappendix II.

WAppendix III.
"Epoxy bond and stop-etch (EBASE) technique enabling backside processing of $(\mathrm{Al}) \mathrm{GaAs}$ heterostructures"

"Unipolar Complementary Bistable Memories Using Gate-Controlled Negative Differential Resistance in a 2D2D Quantum Tunneling Transistor" 


\section{DISCLAIMER}

This report was prepared as an account of work sponsored by an agency of the United States Government. Neither the United States Government nor any agency thereof, nor any of their employees, makes any warranty, express or implied, or assumes any legal liability or responsibility for the accuracy, completeness, or usefulness of any information, apparatus, product, or process disclosed, or represents that its use would not infringe privately owned rights. Reference herein to any specific commercial product, process, or service by trade name, trademark, manufacturer, or otherwise does not necessarily constitute or imply its endorsement, recommendation, or favoring by the United States Government or any agency thereof. The views and opinions of authors expressed herein do not necessarily state or reflect those of the United States Government or any agency thereof. 


\section{DISCLAIMER}

Portions of this document may be illegible electronic image products. Images are produced from the best available original document. 
iii

đ appendix IV.
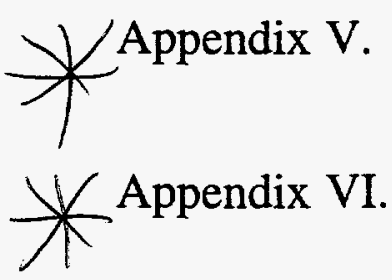

* Appendix VII.

*Appendix VIII.

*Appendix IX.

X Appendix $\mathrm{X}$.

* Appendix XI.

*Appendix XII.

*Appendix XIII

*Appendix XIV

K Appendix XV

Distribution
"Compact Logic/Memory Elements Using a Gated 2D-2D

Quantum Tunneling Transistor"

24

"A Planar Transistor Based on 2D-2D Tunneling in a Double Quantum Well Heterostructure"

29

"Gate Controlled Double Electron Layer Tunneling Transistor (DELTT) and Single Transistor Digital Logic Applications"

65

"Complementary Bistable Memories Using a Novel Double Electron Layer Tunneling Transistor (DELTT)"

74

"Dual-Side Electron Beam Lithography for Independent Submicron Gating of Double Quantum Well Devices"

88

"In-Plane Magnetoresistance Studies of an Extremely Coupled Double Quantum Well"

99

"Observation of Magnetic Breakdown in Double Quantum Wells"

113

"Magnetic Breakdown and Landau-level Spectra of a Tunable Double-Quantum-Well Fermi Surface"

120

"Landau Level Formation and Magnetic Breakdown in Coupled Double Quantum Wells" 141

"Magnetic Breakdown and Landau Level Spectra of a Tunable Double-Quantum-Well Fermi Surface" 148

"Quantum Oscillations of 2-Dimensional to 2-Dimensional Tunneling in Bilayer Electron Gases in Tilted Magnetic Fields"

153

"Magnetoquantum-Resistance Oscillations in TunnelCoupled Double Quantum Wells in Tilted Magnetic Fields: Variable Landau Bi-Ladders"

169

.194

* Removed for separate processing 


\title{
Final Report on LDRD Project:
}

\section{The Double Electron Layer Tunneling Transistor (DELTT)}

\author{
Case Number: 3505.440 \\ Responsible Project Manager: W. B. Gauster, 1112 \\ Principle Investigators: J. A. Simmons, J. S. Moon, M. A. Blount, S. K. Lyo, \\ W. E. Baca, M. E. Sherwin, N. E. Harff, M. V. \\ Weckwerth, J. L. Reno, J. R. Wendt, J. F. Klem, and \\ M. J. Hafich.
}

\section{Purpose of LDRD Research}

The goal of this LDRD was to implement the recently discovered phenomenon of two-dimensional--two dimensional (2D-2D) tunneling in GaAs/AlGaAs double quantum well (DQW) heterostructures as the operating principle for a new type of tunneling device we proposed, the double electron layer tunneling transistor (DELTT). Tunneling has long been considered one of the most promising effects for a new generation of electronic devices, due to (1) its inherently high speed and (2) its possibly increased functionality. The most promising tunneling device to date has been the double barrier resonant tunnel diode (DBRTD). The new 2D-2D tunneling phenomenon, investigated as part of a previous LDRD, offers vastly superior advantages for device possibilities over the 3D-2D tunneling on which traditional DBRTDs are based. These advantages are:

(1) Much sharper tunneling resonances. The controlling factor in electron tunneling is simultaneous conservation of energy $E$ and transverse momentum $\mathbf{k}_{\perp}$. Because the 3D layer in DBRTDs has a continuum of allowed $E s$ for each $\mathbf{k} \perp$ value, tunneling occurs over a broad range of bias conditions, leading to the characteristic saw tooth I-V curve. In 2D-2D tunneling, by contrast, the available $k$-space is much more restricted: each occupied $\mathbf{k} \perp$ state can have only one $E$. The result is that tunneling occurs only at one specific bias, resulting in a single sharp peak, and to first order zero tunneling elsewhere. This bias-dependence should lead to increased functionality and more robust off states.

(2) Ability to modulate with a third terminal. Because the top electron layer is 2D rather than 3D, its density is easily modulated with a metallic surface gate. This allows the tunneling to be switched on and off with a third terminal. By contrast, attempts to attach a third terminal to DBRTDs have either not been successful, as in the case of attempting to electrically contact the central resonant tunneling well, or have required small laterally-defined features which cannot be easily fabricated in the large numbers necessary for integrated circuits. 
(3) Amenable to low-power unipolar complementary logic applications. The bias value required to achieve resonance (the on-state) can be easily adjusted by a shallow surface etch, partially depleting the top QW. Thus normally-on and-normally-off devices can be fabricated on the same chip and with the carriers of the same sign. By hooking two appropriately designed DELTTs together in series, the circuit can be made to only have stable points in the valley currents of the two DELTTs, thus producing complementary behaviour. However, the carriers have the same sign in the two DELTTs, meaning the circuit is unipolar. Thus compromises in speed are avoided, and the growth and fabrication is greatly simplified.

The main thrusts of the project were to (1) build a prototype DELTT, and (2) continue a modest parallel basic research effort on DQW physics issues, with significant theoretical support. For the prototype DELTT, we iteratively addressed the issues of (a) reliable fabrication of separate contacts to emitter and collector, utilizing backgates, (b) design for compatible input and output voltages, and (c) design for high temperature and high speed operation. In the basic physics area, we sought to (a) investigate the possibility of using inter-well transitions as tunable sub-millimeter-wave infrared detectors, (b) examine 1D-1D tunneling in grating-gated and/or split-gated DQW structures; and (c) continue our efforts on the recently observed magnetic field-induced energy level anti-crossing in DQWs.

\section{Accomplishments}

Our work on the DELTT and the physics of tunneling in DQWs resulted in 14 refereed publications and over 14 presentations over the life of the project.Attached to this SAND report as appendices are copies of the refereed publication resulting from this work. In addition, several patent disclosures have been filed, and a patent awarded on the original three-layer DELTT concept. A listing of these publications, presentations, and patent-related documents appears in Appendix I below. Here we give descriptive summaries of our major research accomplishments under this LDRD.

Finally, due to extensive publicity concerning our successful demonstration of the DELTT concept, we have received numerous outside inquiries concerning future collaborative efforts on the DELTT and the FIR detector. These include inquiries from UC Santa Barbara, Princeton University, Ratheon/Texas Instruments, Lockheed-Martin, and Lawrence Livermore National Laboratory. It is expected that work on the DELTT will continue, and will include collaborative efforts with one or more of these institutions.

\section{(1) Development of the EBASE flip-chip processing technique}

A crucial component in the ability to fabricate DELTT structures was the development of a reliable technique for fabricating close-proximity backgates. Separate ohmic contacts to the two individual QWs are acheived by diffusing metallic contacts to both QWs, and then depleting electrons from the QW one does not wish to contact. While depletion etches are possible, depletion gates are more flexible and thus desirable in a laboratory setting. While top gates pose no problem, the backgates necessary for producing contact to the top QW only presented a challenge. The first technique we tried, regrowth of the epitaxial structure over ion-implanted backgates in the semiconductor 
substrate, proved unreliable and exhibited problems with low quality of the regrown material. We then tested a novel flip-chip technique where by the epitaxial layers, after having been processed on the frontside, are then epoxied front-side-down on a host substrate. The original substrate is then removed by a stop etch, revealing a smooth back surface only a few $1000 \AA$ away from the QWs for backside processing. This method, deemed the epoxy-bond-and-stop-etch (EBASE) technique, was delightfully successful, and proved to be highly reliable and robust against temperature cyling.

\section{(2) Initial demonstration of transistor action in a DELTT at $T \leq 4.2 \mathrm{~K}$}

Using the EBASE technique, we fabricated large-area DELTT structures in a DQW having electron densities of $2.0 \times 10^{11} \mathrm{~cm}^{-2}$ and $1.4 \times 10^{11} \mathrm{~cm}^{-2}$, corresponding to a Fermi energy difference of $2.0 \mathrm{meV}$. These structures exhibited excellent electrical characteristics at $4.2 \mathrm{~K}$ and below. At small source-drain biases, the source-drain conductance as a function of control gate voltage exhibited a peak which was 50 times larger than the background conductance. Thus the device exhibited both positive and negative transconductance. In one region, its conductivity dropped as the number of electrons was increased, a novel property unheard of in conventional transistors. In addition, a plot of the source-drain current as a function of source drain voltage at fixed gate voltage exhibited a strong resonant peak. The peak to valley ratio approached 20:1. Finally, the position of the resonant peak in the I-V was shown to be controllable by gate voltage over a wide range. In all cases, the resonant current appeared only when the subbands of the two QWs were aligned, so that electrons could conserve their energy and in-plane momentum in a tunneling event. This dramatic demonstration constituted a clear proof of principle of the DELTT concept.

\section{(3) Demonstration of bistable memory circuits}

In order to show that the DELTT was sufficiently stable and robust for circuit applications, we demonstrated bistable memory circuits using the DELTT. In the first type of circuit, we put a DELTT in series with a resistor so that the circuit had two stable operating points. By pulsing the gate of the DELTT, the circuit could be forced to have only one stable point, either the high or low voltage point depending on the pulse polarity. Upon returning the gate voltage to its idle condition, the circuit remained in the state most recently occupied. Thus the circuit state depended on its past history, demonstrating the memory effect. This also served to demonstrate the multifunctionality of the DELTT, since a similarly behaving conventional NMOS memory requires twice as many components, two transistors and two resistors. Although this demonstration was first performed at $1.5 \mathrm{~K}$, we subsequently also demonstrated the memory at $77 \mathrm{~K}$ using a high temperature DELTT to be described below.

In the second type of memory demonstrated, we hooked two DELTTs together so that the stable operating points lay in the valley current regions of one or the other of the DELTTs. Thus, unlike the single DELTT memory, this allowed the voltage output of the memory to change between states by a factor of 7 , while the current changed by only about $30 \%$. This behavior is similar to that seen in conventional CMOS circuits, which have the advantage of being extremely low power. However, in the case of this DELTT memory the carriers have the same sign in the two transistors, meaning the memory circuit was unipolar. Again, this memory used only half as many transistors as required in a conventional CMOS memory. This circuit, however, has only been demonstrated at $\mathbf{1 . 5}$ 
K.

\section{(4) Design and fabrication of higher temperature DELTTs and demonstration at $77 \mathrm{~K}$}

Upon reflection, we surmised that the maximum temperature at which the DELTT can operate is given when kT is equal to the difference in Fermi energies between the two QWs in the ungated regions between the control gate and the depletion gates. This agreed with our observations of the temperature behavior of the first DELTTs fabricated. To test this, we grew a second DELTT wafer with a considerably larger difference in Fermi energy, $20 \mathrm{meV}$ as opposed to $2 \mathrm{meV}$. This structure exhibited excellent electrical characteristics at $77 \mathrm{~K}$, with peak-to-valley ratios in the I-V of about $6: 1$. Indeed, a 2:1 ratio was exhibited at temperatures of $150 \mathrm{~K}$, and negative differential resistance (NDR) persisted up to $200 \mathrm{~K}$. Using this structure, we also demonstrated the single-DELTT bistable memory at $77 \mathrm{~K}$.

\section{(5) Demonstration of digital logic circuits and oscillators}

We also used the high temperature DELTT to demonstrate additional circuits. Using a single DELTT whose control gate was coupled to two inputs in a resistive summing network, we were able to demonstrate an exclusive-OR (XOR) digital logic function. By applying a bias to the backgate of the DELTT, we showed that the function could be switched to a not-and (NAND) function. By comparison, these two digital logic gates require eight and four transistors respectively when constructed using conventional transistors. Unfortunately, the gain of our circuit was less than one. We expect that with suitable improvements in design the gain can be increased above unity.

A voltage controlled oscillator was also demonstrated, whereby the oscillation inherent in NDR devices was switched on and off by moving the device out of the NDR region with an applied gate voltage.

\section{(6) Extension of EBASE technique to encompass electron-beam lithography}

Although the EBASE technique had worked very well, it was obvious that a very valuable extension would be to make it amenable to mutually aligned electron beam lithography perfomed on both sides of the DELTT structure. This would enable the fabrication of much smaller DELTT devices, resulting in a corresponding decrease in the parasitic capacitances of such structures. Thus the ultimate speed of the DELTT would no longer be limited by such parasitic effects, and the extremely high intrinsic speed of the tunneling process could be approached.

By incorporating a second stop-etch layer, whereby the first, thicker stop etch layer was removed, we were able to use the EBASE process to make epitaxial layers of total thickness 0.23 microns over square centimeter areas. This thickness is so small that the electron beam can easily penetrate it, allowing the same alignment marks to be used for both front side and backside electron beam writing, mutually aligned to one another to

within a few $100 \AA$. Using this technique we fabricated a working DELTT device with submicron gates. 
This was work performed as part of the investitgation of the fundamental physics of double quantum well (DQW) systems. In an earlier LDRD we had demonstrated that when a DQW is subject to in-plane magnetic fields, the Fermi surface breaks into two components, a small lens-shaped orbit due to the upper energy branch, and a larger, hourglass-shaped orbit due to the lower energy branch. Using the temperature dependence of the Shubnikov-de Haas oscillations, we demonstrated that the mass of electrons in the lens orbit was as small as $1 / 3$ the GaAs effective mass, due to the giant distortions of the dispersion curve. Our theoretical predictions indicated that the mass of the electrons in the hourglass orbit should, by contrast, be much as much as a factor of 2 larger than that of GaAs. However, we were previously unable to measure electron masses in the hourglass orbit, due to the fact that their S-dH oscillations are quite weak and so completely obscured by those from the smaller area lens orbit.

To remedy this, we grew an extremely closely coupled DQW sample, such that the symmetric-antisymmetric energy gap was larger than the Fermi energy. As a result, the upper energy branch, or lens orbit, is completely unoccupied at all in-plane magnetic fields. Thus the $\mathrm{SdH}$ oscillations of the hourglass orbit are unobscured and can be easily measured. We found that the mass of electrons in the hourglass orbit is indeed much larger than the GaAs effective mass, consistent with our earlier theoretical predictions.

\section{(8) Magnetic breakdown and complete DOW Landau level spectrum: experiment}

We investigated the energy levels of a double quantum well subject to both in-plane and perpendicular magnetic fields. The in-plane field serves to distort the Fermi surface into a small lens orbit and a larger hourglass orbit, while the perpendicular field causes the formation of a series of Landau levels for each of these two orbits. The system is quite novel, since the electron mass is different for each of the orbits. As a result, when the inplane field is swept at a fixed perpendicular field, the Landau levels of one orbit are depopulated while those of the other become increasingly populated. An extremely challenging measurement of the resistance of two DQW samples as function of both inplane and perpendicular fields was performed. In addition to the two sets of Landau levels mentioned, a third set of Landau levels, whose occupation was independent of inplane field, was also observed. These were found to be due to magnetic breakdown of the Fermi surface, whereby an electron can tunnel in k-space between the lens and hourglass orbits, forming a new circular orbit equivalent to a single undistorted $\mathrm{QW}$. We also performed a simple semiclassical calculation of the Landau level energies, taking into account the changing electron mass of the two orbits. The calculation showed excellent agreement with the data.

\section{(9) Magnetic breakdown and complete DOW Landau level spectrum: theory}

We also developed a full linear response theory of the magnetoresistance of a double quantum well subject to both in-plane and perpendicular magnetic fields. This provided a much more rigorous confirmation of the data we had obtained earlier. The theory used the tight-binding Hamiltonian developed earlier by $\mathrm{Hu}$ and MacDonald to obtain the energy levels, used a phenomenological lineshape for the broadening of the levels, and then applied a linear response theory (Kubo formalism) to obtain the magnetoresistance in the tilted field. The calculations also exhibited evidence of magnetic breakdown, and in general provided good agreement with the data. 
(10) Tunnel-coupled quantum point contacts in split-gate defined double quantum wells

Using our newly developed capability of utilizing electron-beam lithography in conjunction with the EBASE technique, we fabricated a sample consisting of two quantum point contacts (QPCs) on top of one another, defined by mutually aligned splitgates on opposite sides of a double quantum well sample. We were able to demonstrate the independent control over the number of occupied 1D subbands in each of the two QPCs. In addition, we observed evidence for the locking of the two QPCs together in such a fashion that when one depopulates, the other does also. This is attributed to an exchange of charge between the two subbands so as to minimize their total energy; in this way the singularities in the $1 \mathrm{D}$ density of states for each QPC are made to coincide. 
APPENDIX I: List of Refereed Publications and Presentations

\section{Refereed Publications:}

1. M. V. Weckwerth, J. A. Simmons, N. E. Harff, M. E. Sherwin, M. A. Blount, W. E. Baca, and H. C. Chui, 1996. "Epoxy bond and stop-etch (EBASE) technique enabling backside processing of (Al)GaAs heterostructures," Superlattices and Microstructures 20, 561.

2. J. A. Simmons, M. A. Blount, J. S. Moon, W. E. Baca, J. L. Reno, and M. J. Hafich, 1997. "Unipolar Complementary Bistable Memories Using Gate-Controlled Negative Differential Resistance in a 2D-2D Quantum Tunneling Transistor," IEEE Technical Digest of the 1997 International Electron Devices Meeting, Washington DC, Dec. 7-10, p. 755.

3. J. S. Moon, J. A. Simmons, M. A. Blount, W. E. Baca, J. L. Reno, and M. J. Hafich, 1997. "Compact Logic/Memory Elements Using a Gated 2D-2D Quantum Tunneling Transistor," Proceedings of the 1997 International Semiconductor Device Research Symposium, Charlottesville, Virginia, Dec. 10-13, p. 27.

4. J. A. Simmons, M. A. Blount, J. S. Moon, S. K. Lyo, W. E. Baca, J. R. Wendt, J. L. Reno, and M. J. Hafich, 1998. "A Planar Transistor Based on 2D-2D Tunneling in a Double Quantum Well Heterostructure," submitted to Nature.

5. J. S. Moon, J. A. Simmons, M. A. Blount, W. E. Baca, J. L. Reno, and M. J. Hafich, 1998. "Gate Controlled Double Electron Layer Tunneling Transistor (DELTT) and Single Transistor Digital Logic Applications," to appear in Electronics Letters.

6. M. A. Blount, J. A. Simmons, J. S. Moon, W. E. Baca, J. L. Reno, and M. J. Hafich, 1998. "Complementary Bistable Memories Using a Novel Double Electron Layer Tunneling Transistor (DELTT)," to appear in Semiconductor Science and Technology.

7. J. R. Wendt, J. A. Simmons, J. S. Moon, W. E. Baca; M. A. Blount, and J. L. Reno, "Dual-Side Electron Beam Lithography for Independent Submicron Gating of Double Quantum Well Devices," to appear in Semiconductor Science and Technology.

8. M. A. Blount, J. A. Simmons, and S. K. Lyo, 1998. "In-Plane Magnetoresistance Studies of an Extremely Coupled Double Quantum Well," to appear in Phys. Rev. $B$. 
9. N. E. Harff, J. A. Simmons, J. F. Klem, G. S. Boebinger, L. N. Pfeiffer, and K. W. West, 1996. "Observation of Magnetic Breakdown in Double Quantum Wells," Superlattices and Microstructures 20, 595.

10. N. E. Harff, J. A. Simmons, S. K. Lyo, J. F. Klem, G. S. Boebinger, L. N. Pfeiffer, and K. W. West, 1997. "Magnetic Breakdown and Landau-level Spectra of a Tunable Double-Quantum-Well Fermi Surface," Phys. Rev. B. 55, R13405.

11. N. E. Harff, J. A. Simmons, S. K. Lyo, J. F. Klem, G. S. Boebinger, L. N. Pfeiffer, and K. W. West, 1997. "Landau Level Formation and Magnetic Breakdown in Coupled Double Quantum Wells," Physica E 1, 185.

12. J. A. Simmons, N. E. Harff, S. K. Lyo, J. F. Klem, G. S. Boebinger, L. N. Pfeiffer, and K. W. West, 1998. "Magnetic Breakdown and Landau Level Spectra of a Tunable Double-Quantum-Well Fermi Surface," to appear in Physica B.

13. S. K. Lyo, 1998. "Quantum Oscillations of 2-Dimensional to 2-Dimensional Tunneling in Bilayer Electron Gases in Tilted Magnetic Fields," Phys. Rev. B 57, 9114.

14. S. K. Lyo, N. E. Harff, and J. A. Simmons, 1998. "Magnetoquantum-Resistance Oscillations in Tunnel-Coupled Double Quantum Wells in Tilted Magnetic Fields: Variable Landau Bi-Ladders," to appear in Phys. Rev. B.

\section{Presentations}

1. N. E. Harff, J. A. Simmons, J. F. Klem, and M. V. Weckwerth, 1996. "Magnetic Breakdown In Coupled Double Quantum Wells," 1996 March Meeting of the American Physical Society, St. Louis, MO, 3/18-22/96.

2. J. A. Simmons, M. E. Sherwin, M. V. Weckwerth, N. E. Harff, T. M. Eiles, W. E. Baca, H. Q. Hou, and B. E. Hammons, 1996. "Advanced Fabrication Technologies For Nanoelectronics," (INVITED), State of the Art Program on Compound Semiconductors XXIV, Electrochemical Society, Los Angeles, CA 5/5$10 / 96$.

3. N. E. Harff, J. A. Simmons, J. F. Klem, G. S. Boebinger, L. N. Pfeiffer, and K. W. West, 1996. "Observation Of Magnetic Breakdown In Double Quantum Wells," NanoMES '96, Santa Fe, NM, 5/19-24/96.

4. M.V. Weckwerth, J. A. Simmons, N. E. Harff, M. E. Sherwin, M. A. Blount, W. E. Baca, and H. C. Chui, 1996. "Epoxy Bond And Stop-Etch (Ebase) Technique Enabling Backside Processing Of (Al)Gaas Heterostructures," NanoMES '96, Santa Fe, NM, 5/19-24/96. 
5. N. E. Harff, J. A. Simmons, J. F. Klem, G. S. Boebinger, L. N. Pfeiffer, and K. W. West, 1996. "Magnetic Breakdown In Double Quantum Wells," 23rd International Conference on the Physics of Semiconductors, Berlin, Germany, 7/21$26 / 96$.

6. M. A. Blount, J. A. Simmons, N. E. Harff, S. K. Lyo, and M. J. Hafich, 1997. "Magnetoconductance And Effective Mass Of An Extremely Closely Coupled Double Quantum Well, " American Physical Society March Meeting, Kansas City, MO, 3/17-21/97.

7. N. E. Harff, J. A. Simmons, S. K. Lyo, J. F. Klem, G. S. Boebinger, L. N. Pfeiffer, and K. W. West, 1997. "Magnetic Breakdown And Landau Level Spectrum Of Coupled Double Quantum Wells In Tilted Magnetic Fields," American Physical Society March Meeting, Kansas City, MO, 3/17-21/97.

8. J. A. Simmons, W. E. Baca, J. S. Moon, M. A. Blount, and J. R. Wendt, 1997. "Epoxy-Bond-And-Stop-Etch (Ebase) Technique For Close Proximity Submicron Backside Gating Of Algaas/Gaas Heterostructures," American Physical Society March Meeting, Kansas City, MO, 3/17-21/97.

9. N. E. Harff, J. A. Simmons, S. K. Lyo, J. F. Klem, J. R. Wendt, M. A. Blount, W. E. Baca, and T. R. Castillo, 1997. "Electron Transport In Coupled Double Quantum Wells And Wires," Oregon State University (Electrical \& Computer Engineering Dept.), Corvallis, Oregon.

10. M. A. Blount, J. A. Simmons, S. K. Lyo, N. E. Harff, and M. V. Weckwerth, 1997. "Magnetoresistance And Cyclotron Mass In Strongly-Coupled Double Quantum Wells Under In-Plane Magnetic Fields," 24th International Symposium on Compound Semiconductors, San Diego, CA, 9/7-11/97.

11. J. A. Simmons, N. E. Harff. S. K. Lyo, J. F. Klem, G. S. Boebinger, L. N. Pfeiffer, and K. W. West, 1997. "Magnetic Breakdown And Landau Level Spectra Of A Tunable Double Quantum Well Fermi Surface," 12th International Conference on the Electronic Properties of Two-Dimensional Systems, Tokyo, Japan, 9/22$26 / 97$.

12. M. A. Blount, J. A. Simmons, J. S. Moon, W. E. Baca, J. R. Wendt, J. L. Reno, and M. J. Hafich, 1998. "Bistable Memory Cells Utilizing The Double Eletron Layer Tunneling Transistor (DELTT)," 1998 March American Physical Society Meeting, Los Angeles, CA, 3/16-20/98.

13. J. S. Moon, J. A. Simmons, M. A. Blount, W. E. Baca, J. L. Reno, and M. J. Hafich, 1998. "Single Transistor Digital Logic Circuits Based On Gate-Controlled 2d-2d Resonant Tunneling," 1998 March American Physical Society Meeting, Los Angeles, CA, 3/16-20/98. 
14. J. A. Simmons, M. A. Blount, J. .S. Moon, W. E. Baca, J. R. Wendt, J. L. Reno, and M. J. Hafich, 1998. "Double Electron Layer Tunneling Transistor (DELTT) Based On Gate Control Of 2d-2d Tunneling," 1998 March American Physical Society Meeting, Los Angeles, CA, 3/16-20/98. 